\title{
Oxygen-Related Band Gap State in Single Crystal Rubrene
}

\author{
Oleg Mitrofanov, ${ }^{1}$ David V. Lang, ${ }^{2}$ Christian Kloc, ${ }^{1}$ J. Magnus Wikberg, ${ }^{1}$ Theo Siegrist, ${ }^{1}$ \\ Woo-Young So, ${ }^{2}$ M. A. Sergent, ${ }^{1}$ and Arthur P. Ramirez ${ }^{1,2}$ \\ ${ }^{1}$ Bell Labs, Lucent Technologies, Murray Hill, New Jersey 07974, USA \\ ${ }^{2}$ Columbia University, New York, New York 10027, USA \\ (Received 19 May 2006; published 19 October 2006)
}

\begin{abstract}
A molecular exciton signature is established and investigated under different ambient conditions in rubrene single crystals. An oxygen-related band gap state is found to form in the ambient atmosphere. This state acts as an acceptor center and assists in the fast dissociation of excitons, resulting in a higher dark and photoconductivity of oxidized rubrene. The band gap state produces a well-defined photoluminescence band at an energy $0.25 \mathrm{eV}$ below the energy of the $0-0$ molecular exciton transition. Twophoton excitation spectroscopy shows that the states are concentrated near the surface of naturally oxidized rubrene.
\end{abstract}

\section{DOI: 10.1103/PhysRevLett.97.166601}

Charge transport in organic molecular crystals is notably different from inorganic semiconductors. Despite a large number of investigations, understanding the fundamental processes governing charge transport remains a central issue for organic-based devices. In particular, the origin of band gap states and their effect on electronic and optical characteristics are both controversial and important questions $[1,2]$.

Rubrene $\left(\mathrm{C}_{42} \mathrm{H}_{28}\right)$ in single crystal form has emerged as an important aromatic molecular solid, showing the highest field effect transistor (FET) mobility to date among organics $\left(20 \mathrm{~cm}^{2} / \mathrm{Vs}\right)$ [3]. In addition, several studies suggest that bandlike transport is operative [3-6]. Electronic properties in rubrene are, however, highly sensitive to both atmospheric environment as well as material treatment. As is true for inorganic semiconductors, device applications demand the understanding and control of such environmental factors, but the situation in rubrene is far from clear to date. Oxidation of organic crystals often results in modification of transport characteristics [7-11], and recent studies indicate that the presence of ambient oxygen also affects the conductivity of crystalline rubrene [12,13]. Furthermore, chemical analysis of naturally oxidized crystalline rubrene shows that the relative concentration of rubrene peroxide $\left(\mathrm{C}_{42} \mathrm{H}_{28} \mathrm{O}_{2}\right)$ can be as much as $\sim 1 \%$ at a depth of $50 \mathrm{~nm}$ [14], a length scale greater than a typical FET channel depth. Such a high impurity concentration will likely have a significant effect on charge transport, particularly if the impurity forms a band gap state.

In this Letter, we demonstrate the formation of a band gap state in oxidized rubrene crystals and address the impact of the state on charge transport and exciton recombination. A spectral signature consistent with the molecular exciton model is established and investigated under different ambient conditions. A well-defined photoluminescence (PL) band at an energy lower than the highest exciton recombination transition energy develops in the spectra of oxidized rubrene. The PL band reflects an additional radiative recombination path through the oxygen-
PACS numbers: 72.80.Le, 71.20.Rv, 71.35.-y

related state in the band gap. The PL depth profile indicates that the oxygen-related band is emitted only from a surface region. Correlation between the conductivity and luminescence strongly suggests that the oxygen-related state is responsible for the rise in dark and photoconductivity in oxidized rubrene. We propose a model of a deep acceptor state that explains the conductivity and PL characteristics. In addition, this study indicates that the oxygen-related state enhances the dissociation rate for tightly bound molecular excitons.

Rubrene single crystals were grown by the vapor transport method [15] in a stream of pure hydrogen to prevent oxidation during crystal growth (the crystals were handled in air after the growth). Selected crystals were either deliberately oxidized in an oxygen atmosphere for $14 \mathrm{~h}$ at $100{ }^{\circ} \mathrm{C}$ or placed in vacuum shortly after the growth. The latter will be referred to as as-grown crystals. Three groups of samples were studied: (i) as-grown crystals measured in vacuum, (ii) naturally oxidized crystals measured in a normal atmospheric environment, and (iii) thermally oxidized crystals measured in an oxygen environment. PL characteristics were measured using laser $\mathrm{cw}$ excitation $(h \nu=$ $3.815 \mathrm{eV})$ and pulsed two-photon (2P) excitation ( $2 h \nu=$ $3.30 \mathrm{eV})$. Transport measurements were made in a temperature-variable probe station that could be either under vacuum or exposed to various gases.

In organic crystals, PL spectra contain a molecular exciton signature, which is typically modified as compared to the individual molecule spectrum due to the interaction in the crystal. In rubrene, the exciton signature is yet to be established. A pervasive feature of molecular excitons is their strong coupling to molecular vibrations that results in a series of vibronic bands in absorption and PL spectra. The absorption bands in rubrene are regularly spaced by an energy corresponding to the $\mathrm{C}-\mathrm{C}$ stretch vibration mode $\left(\hbar \omega_{\mathrm{C}-\mathrm{C}}=0.177 \mathrm{eV}\right)$ similar to rubrene molecules in solid solutions [16]. The $0-0$ absorption band in single crystals is located at $E_{0-0}^{*}=2.32 \mathrm{eV}(535 \mathrm{~nm})$ (Fig. 1). Higher order vibronic bands are regularly spaced at 
$E_{0-n}^{*}=E_{0-0}^{*}+n \hbar \omega_{\mathrm{C}-\mathrm{C}}$, where $n$ is an integer. PL spectra, on the other hand, show a more complex structure, where the vibronic bands are not evident at room temperature. Only low temperature PL spectra of crystalline rubrene held in vacuum clearly show the vibronic structure of exciton transitions shifted to lower energies compared to the absorption transitions. The first luminescence band (band I, letter notations are introduced to differentiate PL bands throughout the text) is located at $2.18 \mathrm{eV}(570 \mathrm{~nm})$, $0.14 \mathrm{eV}$ below the $0-0$ absorption line (Fig. 1). A series of weaker vibronic bands can be observed at energies $E_{0-n}=$ $E_{0-0}-n \hbar \omega_{\mathrm{C}-\mathrm{C}}(2.00$ and $1.83 \mathrm{eV})$.

At a finite temperature, the PL spectrum reveals a substantial change in the exciton recombination process. The intensity of band I decreases by at least 3 orders of magnitude as the temperature increases from $\sim 20$ to $300 \mathrm{~K}$. The vibronic bands decrease similarly in intensity as shown in the inset in Fig. 1. Along with the PL quenching at higher temperatures, additional radiative transitions appear at lower energies [Fig. 2(a)]. At $T>70 \mathrm{~K}$, a new band (II) develops at $2.11 \mathrm{eV}$ with a vibronic band at $1.93 \mathrm{eV}$. The PL intensity of this series varies with temperature in a similar fashion as series I. At higher temperatures $(T>100 \mathrm{~K})$, another band (III) appears at $2.05 \mathrm{eV}$, and it dominates in the spectrum up to $T=300 \mathrm{~K}$. Defining the bands that reflect the exciton relaxation process in as-grown rubrene is essential, because the oxygen-related defect produces emission at similar energies, as we will show below.

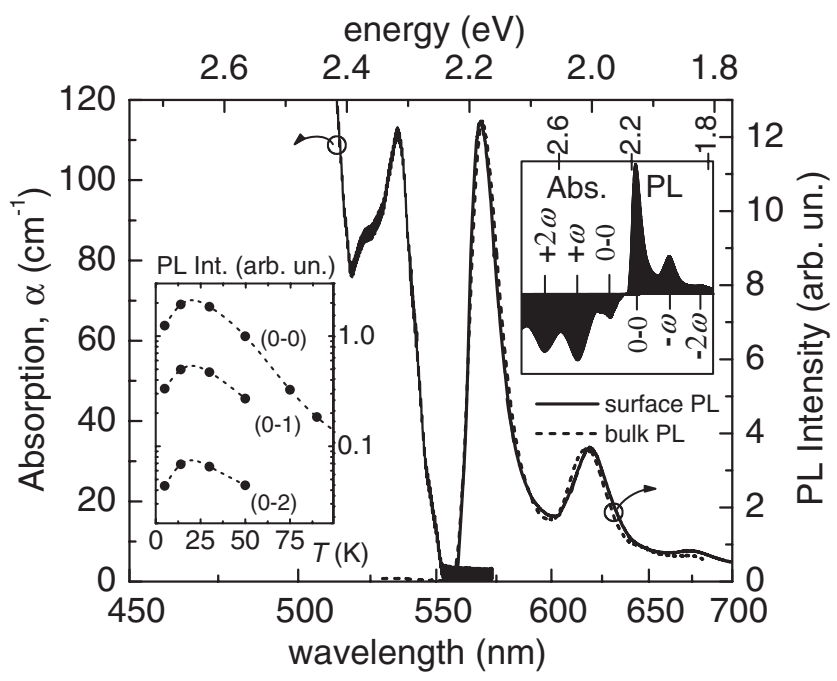

FIG. 1. A part of the absorption spectrum showing the lowest energy molecular exciton transition (0-0) and the PL spectra of crystalline rubrene at $20 \mathrm{~K}$ in vacuum. An inset in the upper right corner shows the relation between the absorption and the PL peaks. The surface luminescence spectrum is measured after $\mathrm{cw}$ excitation $(h \nu=3.815 \mathrm{eV}$, the penetration depth $d \sim 1 \mu \mathrm{m})$ and the bulk spectrum is measured after $2 \mathrm{P}$ excitation $(100 \mathrm{fs}$, $h \nu=1.65 \mathrm{eV}, \mathrm{NA}=0.3$ ) at a depth of $100 \mu \mathrm{m}$ from the surface. An inset in the lower left corner shows the temperature dependence of intensity of the main band I and two higher order vibronic bands, corresponding to transitions (0-1) and (0-2).
First, we confirm that the observed bands are the characteristic features of as-grown rubrene. Prior work [14] established the presence of rubrene peroxide on the surface of naturally oxidized samples. Thus, in order to characterize the material unperturbed by oxidation, PL spectra must be taken from a depth substantially exceeding the oxygen diffusion length. Optical excitation deep in the crystal $(>1 \mu \mathrm{m})$ is achieved through the process of $2 \mathrm{P}$ absorption. Low-intensity $100 \mathrm{fs}$ laser pulses with a photon energy well below the absorption edge of rubrene, $h \nu=1.65 \mathrm{eV}$, propagate freely in the crystal. The pulses generate excitons only in a small region in the focus of the laser beam. Because the luminescence bands of crystalline rubrene lie in the transparent region (Fig. 1), the emitted photons are not reabsorbed in the crystal. The PL characteristics measured using the $2 \mathrm{P}$ excitation in the bulk and one-photon excitation on the surface of as-grown rubrene samples kept in vacuum show the same structure (Fig. 1) and the same temperature dependence. These bands correspond to optical transitions in as-grown rubrene.

We next address an impurity-related signature in the PL spectra. In contrast to the vacuum-held samples, discussed above, samples kept in air show additional optical transitions. For these measurements, the PL spectra were obtained on crystals sealed in a quartz capsule containing oxygen at normal pressure. The sealed capsule was heated at $100{ }^{\circ} \mathrm{C}$ for $14 \mathrm{~h}$ prior to measurement to enhance the oxidation process. Similar to as-grown samples, the series of vibronic bands $I$ is present in the PL spectra at low

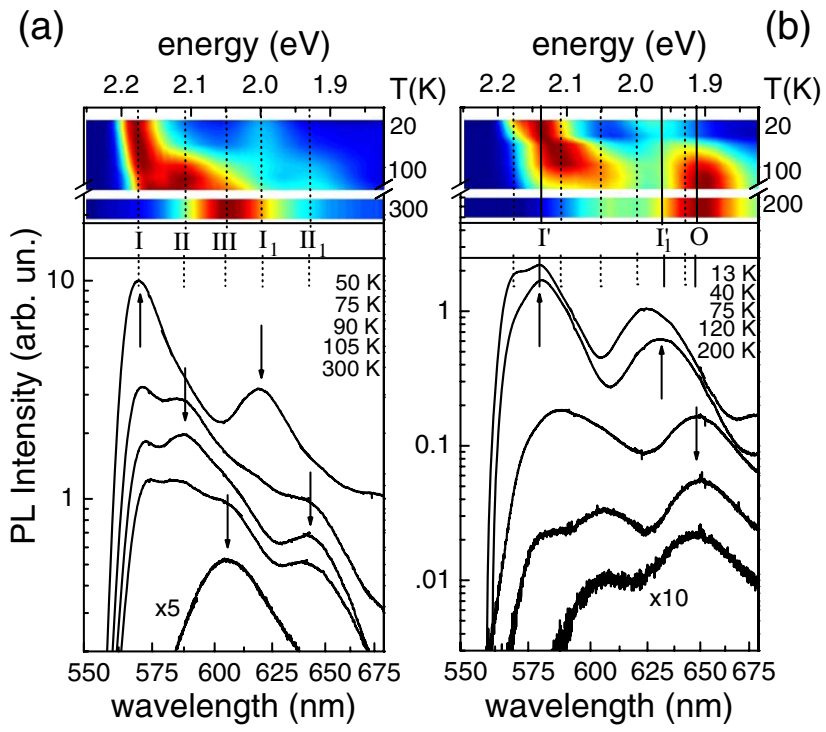

FIG. 2 (color online). PL characteristics of (a) as-grown and (b) oxidized rubrene crystals at different temperatures. The asgrown sample is held in vacuum, and the oxidized sample is sealed in a capsule containing oxygen at a normal pressure. PL bands I, II, and III, and their higher order vibronic bands $I_{1}$ and $\mathrm{I}_{2}$, characteristic of as-grown samples are indicated by arrows in (a), PL band $\mathrm{I}^{\prime}$, vibronic band $\mathrm{I}_{1}^{\prime}$, and band $\mathrm{O}$ characteristic of oxidized samples are indicated by arrows in (b). The color maps show spectral characteristics normalized for every temperature. 
temperatures [Fig. 2(b)]. However, the intensity of the series is reduced relative to as-grown crystals, and an additional series of bands ( $\left.\mathrm{I}^{\prime}\right)$ shifted by about $40 \mathrm{meV}$ is clearly seen. As the temperature increases to $\sim 70 \mathrm{~K}$, in addition to band II, present in the spectra of the vacuumheld samples, strong emission at $1.92 \mathrm{eV}$ appears in the spectrum. The new band $(\mathrm{O})$ should not be confused with the first phonon replica $\mathrm{II}_{1}$ located at $1.93 \mathrm{eV}$, the intensity of which follows the intensity decrease of band II as the temperature rises. Band $\mathrm{O}$ remains the main spectral signature of the oxidized samples at temperatures above $200 \mathrm{~K}$, while band III, which dominates the PL spectra of as-grown rubrene, is strongly suppressed. Band $\mathrm{O}$ is also present in the PL spectra of naturally oxidized rubrene crystals. A summary of the optical transitions in as-grown and oxidized rubrene is presented in Table I.

A clear demonstration of surface oxidation is shown in Fig. 3 in the room temperature PL characteristics measured at various depths of a naturally oxidized (several weeks) rubrene crystal held in air. Band $\mathrm{O}$ is clearly the strongest band in the PL spectra measured when the excitation spot is positioned on the surface $(z=0)$. The intensity of this band decreases as the excitation spot is translated into the crystal. Band III, weaker on the surface, gradually increases inside the crystal. Band I, present in the surface spectrum, decreases in the bulk; however, this decrease is attributed to reabsorption of emission inside the crystal due to the fact that the absorption tail of the 0-0 transition is substantially broadened at room temperature. For the single-photon excitation, limited to a depth of $\sim 1 \mu \mathrm{m}$, the PL spectrum of the oxidized sample in air shows emission in band $\mathrm{O}$, while an as-grown sample shows emission in band III. The oxygen-related impurity is clearly concentrated near the surface.

We argue that an oxygen-related state provides a recombination path for molecular excitons resulting in emission in band $\mathrm{O}$ positioned about $0.25 \mathrm{eV}$ below the lowest $0-0$ transition of band I. Consequently, the impurity state quenches the radiative recombination processes observed in as-grown rubrene. The oxygen-related band gap state has several possible origins: (1) a rubrene molecule perturbed by the presence of a neighboring oxygen molecule; (2) a rubrene peroxide molecule formed after reaction with a molecular oxygen; (3) a rubrene molecule perturbed by the presence of a neighboring rubrene peroxide molecule.

TABLE I. Photoluminescence data of crystalline rubrene: transition energy $E$ and the shift $\hbar \omega$ of the first vibronic band.

\begin{tabular}{cll}
\hline \hline & $E(\mathrm{eV})$ & $\hbar \omega(\mathrm{eV})^{\mathrm{a}}$ \\
\hline I & 2.18 & 0.18 \\
II & 2.11 & 0.18 \\
III & 2.05 & \\
I' $^{\prime}$ & 2.14 & 0.18 \\
O & 1.92 & \\
\hline \hline
\end{tabular}

${ }^{\text {a }}$ The presence of the higher order vibronic bands for bands III and $\mathrm{O}$ is not determined due to broadening of the bands.
The above data associate a distinct PL feature with an oxygen-related state. Distinct effects in the material's conductivity have also been attributed to the presence of oxygen $[12,13]$. Indeed, we have found that the dark and photoconductivity of rubrene crystals oxidized using the same procedures as for the PL measurements is dramatically increased relative to as-grown rubrene [17]. Currentvoltage characteristics were measured using evaporated coplanar wide gold electrodes with various gaps $l$ from 25 to $150 \mu \mathrm{m}$. A typical increase of the dark and photoconductivity in oxidized samples is shown in Fig. 4. The charge neutrality dictates that the dark current increase (due to holes [4,17]) is caused by the creation of negatively charged centers (acceptors) due to the oxygen treatment. Below, we propose a consistent explanation that ties together both the transport and PL signature of oxygen incorporation in rubrene.

We first consider the impact of the acceptor state on the photoconductivity. It is currently debated whether the optical excitation in rubrene results in generation of weakly bound charge transfer excitons [6], which can dissociate at room temperature, or in excitation of tightly bound molecular excitons, which require a complex dissociation mechanism. Pronounced molecular vibronic bands in the PL spectrum suggest that photogenerated carriers are initially tightly bound in the form of molecular excitons that couple to the molecular vibration. In tetracene, where the structure of the $\pi$ orbital is similar to that of rubrene, the exciton binding energy is $\sim 400 \mathrm{meV}$ [18]. A similar binding energy might be expected in rubrene. The exciton dissociation process, therefore, is likely to involve defector impurity-assisted exciton dissociation mechanisms. Although the high binding energy results in wave function confinement, the excitons are nevertheless free to move to neighboring molecules. An electron can be trapped at a defect site, thus releasing a free hole (and increasing the photoconductivity). The trapping process provides an al-

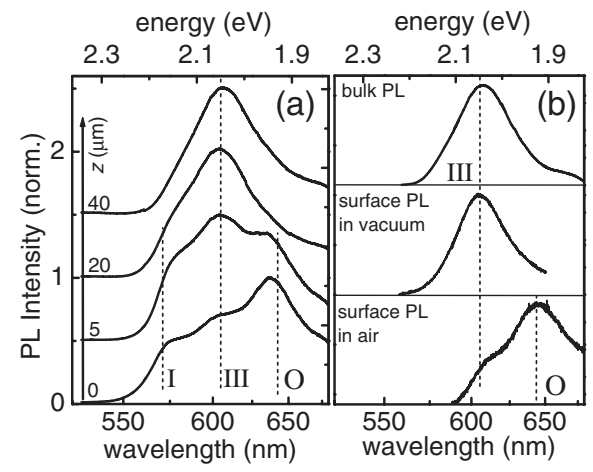

FIG. 3. (a) Normalized room temperature PL characteristics measured using $2 \mathrm{P}$ excitation (100 fs, $h \nu=1.65 \mathrm{eV}, \mathrm{NA}=0.8$ ) at various depths $z$ inside a crystalline rubrene sample naturally oxidized in air. (b) The bulk rubrene spectrum (top) measured with NA $=0.3$ at $z=100 \mu \mathrm{m}$ is compared to normalized room temperature PL characteristics of a vacuum-held sample (center) and an oxidized sample after single-photon excitation (bottom). 


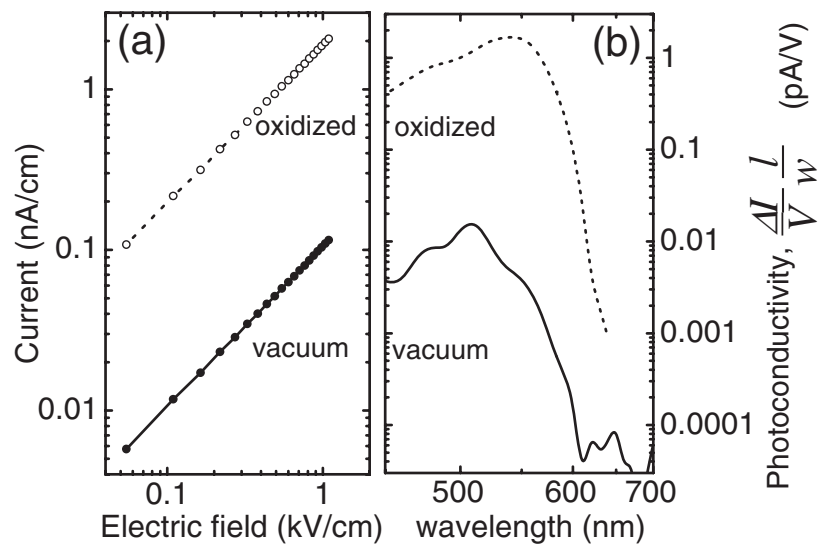

FIG. 4. (a) Dark current-voltage characteristics and (b) photocurrent spectral response of an oxidized rubrene sample and a sample held in vacuum (solid line). The photocurrent $\Delta I=$ $I_{\text {photo }}-I_{\text {dark }}$ is measured using a grating monochromator and a broadband white light source modulated at $10 \mathrm{~Hz}$.

ternative relaxation mechanism and causes exciton PL quenching. The trapping events can also be reflected in the PL spectrum as a new band, such as the band $\mathrm{O}$ that we observe.

Evolution of the PL characteristics and photocurrent with temperature in a system where defects provide additional recombination paths for strongly bound excitons thus depends on exciton diffusion rather than thermally activated exciton dissociation. As the exciton diffusion length increases with temperature and any potential barriers to trapping at defects are more easily surmounted, recombination through defect states becomes more efficient and unassisted radiative exciton recombination decreases. A correlation between the time evolution of PL and photocurrent after a pulsed laser excitation was recently shown in naturally oxidized rubrene [6]. In this study, the photocurrent reaches a maximum within several microseconds after the excitation, while the photoluminescence intensity exponentially decreases within the same time interval. This is consistent with our view that molecular excitons dissociate at defects and release only free holes as a result, not electron-hole pairs.

Based on these experimental observations, we propose the following explanation of the dark and photoconductivity enhancement in oxidized rubrene. From the shift of the $\mathrm{O}$ band relative to band I, we suggest that an acceptor level is formed $\sim 0.25 \mathrm{eV}$ above the valence band as a result of oxidation. In equilibrium, some of these acceptor centers capture electrons, leaving free holes in the valence bandi.e., oxidation results in the $p$-type doping of rubrene. In the event of optical excitation, generated excitons quickly decay to the lowest excited singlet state. The exciton recombines radiatively or, in the presence of the neutral acceptor site, the electron decays into the acceptor level, and the remaining hole acquires sufficient kinetic energy to overcome the binding barrier and becomes free. It participates in charge transport until it is captured on the accep- tor level. The presence of the acceptor defect, therefore, assists in exciton dissociation and the increase of photoconductivity, while thermal activation of the acceptors results in higher dark conductivity.

Substantial experimental evidence suggests that crystal defects and impurities are responsible for the conductivity enhancement in crystalline rubrene. A PL band $(\mathrm{O})$ is associated with the oxygen-related band gap state, which forms in an atmospheric environment and increases the dark and photoconductivity of rubrene. Control of the oxygen impurity may open possibilities for the modification of rubrene properties.

This work was partially supported by the U.S. Department of Energy (No. DE-FG02-04ER46118), the Nanoscale Science and Engineering Initiative of the National Science Foundation (No. NSF-CHE-0117752), and by the New York State Office of Science, Technology and Academic Research (NYSTAR).

[1] D. V. Lang, X. Chi , T. Siegrist, A. M. Sergent, and A.P. Ramirez, Phys. Rev. Lett. 93, 086802 (2004).

[2] C. Goldmann, C. Krellner, K. P. Pernstich, S. Haas, D. J. Gundlach, and B. Batlogg, J. Appl. Phys. 99, 034507 (2006).

[3] V. Podzorov, E. Menard, A. Borissov, V. Kiryukhin, J. A. Rogers, and M.E. Gershenson, Phys. Rev. Lett. 93, 086602 (2004).

[4] V. Podzorov, E. Menard, J. A. Rogers, and M.E. Gershenson, Phys. Rev. Lett. 95, 226601 (2005).

[5] O. Ostroverkhova, D. G. Cooke, F. A. Hegmann, J. E. Anthony, V. Podzorov, M.E. Gershenson, O.D. Jurchescu, and T. T. Palstra, Appl. Phys. Lett. 88, 162101 (2006).

[6] H. Najafov, I. Biaggio, V. Podzorov, M. F. Calhoun, and M. E. Gershenson, Phys. Rev. Lett. 96, 056604 (2006).

[7] A. G. Chynoweth, J. Chem. Phys. 22, 1029 (1954).

[8] O. D. Jurchescu, J. Baas, and T. T. M. Palstra, Appl. Phys. Lett. 84, 3061 (2004).

[9] A. Vollmer, O. D. Jurchescu, I. Arfaoui, I. Salzmann, T. T. M. Palstra, P. Rudolf, J. Niemax, J. Pflaum, J.P. Rabe, and N. Koch, Eur. Phys. J. E 17, 339 (2005).

[10] O. D. Jurchescu, J. Baas, and T. T. M. Palstra, Appl. Phys. Lett. 87, 052102 (2005).

[11] S. Ogawa, T. Naijo, Y. Kimura, H. Ishii, and M. Niwano, Appl. Phys. Lett. 86, 252104 (2005).

[12] V. Podzorov, V. M. Pudalov, and M.E. Gershenson, Appl. Phys. Lett. 85, 6039 (2004).

[13] T. Takahashi, T. Takenobu, J. Takeya, and Y. Iwasa, Appl. Phys. Lett. 88, 033505 (2006).

[14] D. Kafer and G. Witte, Phys. Chem. Chem. Phys. 7, 2850 (2005).

[15] R. A Laudise, C. Kloc, P. G. Simpkins, and T. Siegrist, J. Cryst. Growth 187, 449 (1998).

[16] T. T. Nakashima and H. W. Offen, J. Chem. Phys. 48, 4817 (1968).

[17] W.-Y. So et al. (to be published).

[18] K. Hummer and C. Ambrosch-Draxl, Phys. Rev. B 71, 081202(R) (2005). 